\title{
Normes pour les bibliothèques et les services d'information au sein des établissements de soins de santé au Canada - 2006
}

Les normes qui suivent, fondées sur les résultats, se veulent un guide de structure pour les services de bibliothèque au sein d'un établissement de soins de santé.

\section{Crédits}

L'Association des bibliothèques de la santé du Canada / The Canadian Health Libraries Association (ABSC / CHLA) tient à remercier l'Association des bibliothèques médicales (Medical Library Association) de l'autorisation accordée pour l'utilisation de la publication de la MLA : Standards for Hospital Libraries 2002, incluant les révisions de l'année 2004 [1].

\section{Introduction}

L'accès efficace en temps opportun à de la documentation sur la santé fondée sur les résultats constitue un élément important de la prestation de soins sécuritaires auprès des patients. Prenant en compte la complexité et les contraintes de temps qu'impose la prestation des soins, la localisation de l'information adéquate au moment voulu devient de plus en plus difficile pour plusieurs praticiens de la santé. Un service solide de bibliothèque et d'information crée le lien entre les résultats et les décisions de traitement du patient [2-4].

Les besoins en information et en services de bibliothèque sont reconnus par les normes d'organismes d'agrément tels que le Conseil canadien d'agrément des services de santé (CCASS), le Liaison Committee on Medical Education (LCME) et les programmes de résidence et d'agrément du Collège royal des médecins et chirurgiens du Canada.

L'évolution des technologies, la régionalisation, l'informatique et les nouvelles approches aux soins de santé ont provoqué d'importants changements aux établissements de soins de santé au cours des années, comme on le constate à la lecture des normes révisées de 2003 du CCASS basées sur son programme MIRE : mesures implantées pour le renouveau de l'évaluation. Les normes de 1995, les plus récentes pour les bibliothèques de la santé, pour les services d'information et de bibliothèque au sein des établissements de santé au Canada, doivent être revisées.

Avant de décider de récrire ces normes à partir des normes 2002 substantiellement révisées de la Medical Library Association (MLA), le Groupe de travail a passé en revue les normes d'autres bibliothèques. Les lignes directrices de 2000 de l'Australian Library and Information Association (ALIA) for
Australian Health Libraries sont en grande partie basées sur les normes canadiennes de 1995 et sont présentement en révision. Elles en ont la même structure et le même format. Les normes du Irish Health Sciences Librarians Group for Irish Healthcare Library and Information Services, $2^{\mathrm{e}}$ édition 2005 viennent tout juste d'être publiées; elles sont elles aussi basées en grande partie sur les normes canadiennes de 1995 et sur les normes australiennes. Cependant, les normes de la MLA ont été retenues pour leur disposition, leur clarté et leur convivialité.

\section{Norme $n^{\circ} 1$ - Administration et organisation}

Le service de bibliothèque et d'information dispose de son propre budget et le ou la bibliothécaire des sciences de la santé, à titre de directeur ou directrice du service, relève de la haute direction. Le service de bibliothèque et d'information est intégré aux services de développement et de la prestation de services destinés à répondre aux besoins de l'organisme en connaissances et en information basées sur les résultats et est un collaborateur clé en ce qui concerne quelque initiative de gestion de l'information.

\section{Objectif visé}

La norme 5.3 du Conseil canadien d'agrément des services de santé exige que l'organisme mette à la disposition de son personnel, des clients et des familles de la documentation de référence de recherche d'information, alors que la norme 5.4 exige que

- Des systèmes soient en place pour offrir une direction claire et un accès en temps opportun à l'éducation, aux références et à la documentation de recherche, tous évalués en rapport avec les besoins actuels et futurs

- Une méthode soit mise en place pour assurer le lien aux bases de données externes pertinentes, aux réseaux et organismes de recherche

Afin que ces exigences soient respectées, la bibliothèque et le service d'information doivent être en mesure de communiquer avec les décideurs aux plus hauts niveaux de l'établissement. Le statut du service facilite ce processus et accroît la capacité de la bibliothèque à collaborer avec le personnel de tous les domaines au sein de l'hôpital ou de la région sanitaire. 


\section{Norme n 2 - Gestion}

Le service de bibliothèque et d'information est géré par un ou une bibliothécaire professionnel(le) qui possède une maîtrise en bibliothéconomie et en sciences de l'information d'une université ou d'un programme reconnu par l'American Library Association (ALA).

\section{Objectif visé}

Un ou une bibliothécaire professionnel(le) a obtenu une maîtrise dans le cadre d'un programme agréé par l'ALA ou par un organisme national approprié.

Les compétences exigées des bibliothécaires professionnels comprennent une connaissance approfondie des ressources imprimées et électroniques, de même que de la conception et de la gestion de services d'information qui répondent aux besoins spécifiques d'information des individus ou groupes desservis [5-7].

Le rôle du ou de la bibliothécaire professionnel(le) est comme suit :

- Fournir des services de référence et de recherche à l'appui de l'administration de la médecine, des services infirmiers et des disciplines connexes en santé

- Promouvoir les connaissances en information et le développement des compétences des clients en information par le biais de formation formelle et informelle

- Assurer la satisfaction de la clientèle en surveillant les demandes de références et de recherche et par l'évaluation du rendement de la bibliothèque et des indicateurs de qualité

- S'assurer que les collections de la bibliothèque reflètent les besoins d'information du milieu en perpétuel changement des soins de santé

- Assurer une stratégie et une direction au service

- S'assurer que le travail de la bibliothèque et du service d'information reflète les objectifs stratégiques de l'établissement et qu'il soit centré sur une prestation de services efficace et efficiente

- Participer aux initiatives de gestion stratégique

- Assurer un service centré sur le client par le biais d'une gestion des ressources humaines efficace

- S'assurer de la conformité aux réglementations externes

- Gérer les finances de façon responsable en ce qui concerne le personnel, les opérations et les actifs de l'établissement

- Évaluer les nouvelles technologies de l'information et mesurer leurs implications pour les services de bibliothèque

- Négocier des ententes de licences auprès de fournisseurs de ressources de connaissances basées sur les résultats

\section{Norme $n^{\circ} 3$ - Dotation de personnel}

Le service de bibliothèque et d'information est doté en personnel conformément à la formule de dotation d'établissement polyvalent de la MLA - FTE/700 [1] :

- Si le résultat est inférieur ou égal à $1 \mathrm{FTE}$, un pourcentage de 1 FTE doit être un ou une bibliothécaire professionnel(le). Il peut s'agir d'un poste de bibliothécaire contractuel(le), itinérant(e) ou d'une entente de coentreprise. Plus de détails à ce sujet se trouvent à l'Annexe 1

- Si le résultat est de 1-3 FTE, alors 1 FTE doit être un ou une bibliothécaire professionnel(le)
- Si le résultat est plus de 3 FTE, $33 \%$ des postes doivent être des postes de professionnels et le poste de directeur du service doit être comblé par un ou une bibliothécaire professionnel(le) [8]

Les pourcentages restants doivent être un mélange de techniciens qualifiés en bibliothéconomie, d'assistants et de commis.

\section{Objectif visé}

Une bibliothèque à court de personnel ou dotée de personnel sous-qualifié ne peut pas répondre aux besoins d'information basée sur les connaissances ni de ceux d'un milieu misant sur l'information fondée sur les résultats. La demande en information est tributaire de l'envergure et de la complexité de l'établissement ainsi que des facteurs suivants :

- Le nombre de résidents en médecine, d'étudiants sousgradués en médecine et d'internes d'autres programmes de l'établissement

- L'étendue géographique de la région et l'ampleur de la régionalisation de la région concernée. Les bibliothèques en santé régionales totalement intégrées offrent des services non seulement aux établissements de soins de courte durée mais aussi à ceux de soins de longue durée et de soins prolongés, de santé communautaire et de services de soins médicaux d'urgence, souvent répartis dans des régions géographiques étendues

La formule de la MLA offre une méthode facile pour le calcul des ratios en personnel. Elle s'appuie sur des étalons établis pour les institutions américaines et canadiennes [9].

Pour obtenir plus de détails sur cette norme, veuillez vous reporter à la Feuille d'information de l'ABSC : Planification des ressources humaines pour les bibliothèques au sein des établissements de santé, juillet 2005 (http://www.chlaabsc.ca/member/anterior/docs/fs23.pdf).

\section{Norme n' 4 - Services}

Le ou la bibliothécaire assure une évaluation permanente des besoins des services d'information de l'établissement et utilise cette évaluation aux fins de l'élaboration et de la mise en œuvre des services appropriés qui répondent à ces besoins et qui maintiennent l'harmonisation avec la mission de l'établissement, sa vision, ses objectifs et sa planification stratégique.

Les services doivent comprendre

- L'accès convivial à la recherche experte

- La formation sur l'utilisation des ressources électroniques et imprimées basées sur la connaissance

- La formation en recherche basée sur les résultats

- Une technologie adéquate qui assure l'accès aux ressources électroniques

- Un catalogue des ressources qui permet un accès et une acquisition efficaces en matière de documentation.

- La livraison de documentation non disponible dans les collections électroniques ou imprimées de la bibliothèque

\section{Objectif visé}

Les méthodes de prestation de services d'information basée sur les connaissances sont en perpétuel changement. Le ou la bibliothécaire doit constamment évaluer ces nouvelles méthodes afin de s'assurer que les services offerts par la bibliothèque reflètent les besoins des groupes d'utilisateurs. 


\section{Norme n 5 - Ressources}

Le service de bibliothèque et d'information offre des ressources basées sur la connaissance à l'appui de tous les besoins d'information des utilisateurs, y compris, sans s'y limiter, des ressources en soins des patients, de formation, d'administration, de recherche, de santé communautaire et en programmes d'approche. Le ou la bibliothécaire utilise une variété d'outils et de techniques, tant formels qu'informels, pour évaluer les besoins basés sur les connaissances du personnel médical et du personnel en soins de santé.

\section{Objectif visé}

Les ressources sont évaluées en comparaison avec les listes standard de collections, de publications d'experts du domaine, de collections des autres bibliothèques et de sondages auprès des utilisateurs.

\section{Ligne directrice}

Les ressources peuvent comprendre

- Collections de ressources imprimées, électroniques et multimédia qui font autorité et qui permettent la fourniture en temps opportun d'information basée sur les connaissances

- Consortiums d'ententes ou d'adhésion à des bibliothèques d'information qui permettent la fourniture efficace de documents non disponibles sur les lieux

\section{Norme $n^{\circ} 6$ - Promotion}

La bibliothèque et le service d'information font la promotion active de services d'information basée sur les connaissances auprès de tous les principaux groupes de clients.

\section{Objectif visé}

Le ou la bibliothécaire publie les services et les ressources afin d'augmenter le niveau de sensibilisation des utilisateurs et d'accroître l'utilisation efficace des services et des ressources offertes. La promotion efficace de la bibliothèque informe les utilisateurs de la disponibilité des bases de données et des ressources offertes et élimine les barrières tout en encourageant l'utilisation accrue d'information basée sur les résultats pour les décisions relatives au traitement.

\section{Ligne directrice}

La documentation et les programmes promotionnels peuvent comprendre, sans s'y limiter, ce qui suit :

Bulletins

Participation à des comités

Babillards électroniques

Célébrations d'une semaine ou d'un mois consacré à la bibliothèque

Journées portes ouvertes

Livrets d'information, brochures et sommaires d'orientation

\section{Présentations}

Inclusion d'information sur la bibliothèque dans les trousses d'orientation destinées au nouveau personnel et aux nouveaux résidents

\section{Norme n 7 - Législation et conformité}

Le service de bibliothèque et d'information se conforme à la législation applicable, aux codes de déontologie de l'ABSC / CHLA et de la MLA (http://www.chla-absc.ca/ assoc/abscwhat.html\#code) ainsi qu'aux politiques, normes et procédures de l'établissement.

\section{Norme $n^{\circ} 8$ - Accessibilité}

Les ressources basées sur les connaissances sont accessibles tous les jours, 24 heures sur 24 .

\section{Objectif visé}

Puisque les décisions cliniques se prennent souvent endehors des heures normales de travail, les ressources en information basée sur les connaissances doivent être accessibles en tout temps. Selon l'envergure et la complexité de l'institution, cela peut vouloir dire que

- Les ressources sont accessibles par le biais de l'intranet de l'établissement ou via Internet

- Des mécanismes sont en place qui assurent l'accès à la bibliothèque après les heures normales de travail

\section{Norme n 9 - Environnement}

La bibliothèque et le service d'information jouissent d'un environnement approprié à la fourniture d'information basée sur les connaissances. L'espace physique répond aux exigences actuelles et futures (pour une période de trois à cinq ans) propres aux ordinateurs en réseau, aux collections imprimées, aux postes de travail pour le personnel, de même qu'aux exigences d'espaces propices à l'étude dans une atmosphère de tranquillité et d'espaces de réunion en groupe [10]. Les équipements et les espaces pour la tenue d'ateliers sont à la disposition des services de la bibliothèque.

\section{Objectif visé}

Malgré le volume d'information actuellement offert par les moyens électroniques, « les bibliothèques continueront à offrir des espaces d'étude pour des groupes de travail et des individus engagés dans une démarche exigeant la tranquillité, et des espaces pour la formation en utilisation des ressources d'information. Les utilisateurs exigent des espaces qui soient confortables pour la réflexion et le travail, qui demandent de passer de longues périodes devant des écrans, et des systèmes d'aide et d'assistance sur place, de même que des appareils périphériques de qualité tels que des imprimantes et des écrans à haute résolution. Le furetage sera une des fonctions essentielles des bibliothèques. Une mixité d'espaces d'étude pour les groupes et les individus, des points de service polyvalents destinés à aider les utilisateurs tant au niveau technologique qu'au niveau des ressources d'information, davantage d'accent sur l'information en santé communautaire, ainsi que des espaces plus attrayants font tous partie des caractéristiques » [11].

Plusieurs formules de calcul peuvent être utilisées pour déterminer les estimations de besoins d'espace pour la bibliothèque. Le Guide de gestion des bibliothèques en santé de la MLA (Guide to Managing Health Care Libraries) offre un excellent tableau qui résume les options de calcul 
d'espace et les autres exigences de la bibliothèque en ce qui a trait aux charges de plancher [10].

\section{Références}

1. Hassig RA, Balogh L, Bandy M, Doyle JD, Gluck JC, Lindner KL, et al. Standards for hospital libraries 2002 incluant les revisions de 2004. J Med Lib Assoc. 2005 Apr;93(2):282-283.

2. Institute of Medicine. Committee on Quality of Health Care in America. Crossing the quality chasm : a new health system for the 21st century. Washington, D.C.: National Academy Press; 2001.

3. Summerskill W. Literature searches: look before you leap. Lancet. 2005 Jul 2-8;366(9479):13-14.

4. Williams L, Zipperer L. Improving access to information: librarians and nurses team up for patient safety. Nurs Econ. 2003 Jul-Aug;21(4):199-201.

5. Alliance of Libraries, Archives and Records Management. Competency Profile. Informaiton Resources Management specialists in Archives, Libraries and Records Management: A comprehensive Cross-Sectoral competency Analysis. 1999 April 1999.

6. Crosby O. Librarians: Information experts in the information age. OOQ. 2001(Hiver 2000-01):2.

7. Medical Library Association. Medical Librarianship: A Career Beyond the Cutting Edge.

8. Creth SD, Duda F. Personnel administration in libraries. 2nd ed. New York: Neal-Schuman Publishers; 1989.

9. MLA Benchmarking Network. The composite hospital library : 2001 benchmarking aggregate data tables. 2002.

10. Holst R, Phillips SA, Bensing KM, Medical Library Association. The Medical Library Association guide to managing health care libraries. New York: Neal-Schuman Publishers; 2000.

11. Ludwig L, Starr S. Library as place: results of a delphi study. J Med Libr Assoc. 2005 Jul;93(3):315-326.

\section{Annexe 1}

Lorsque le résultat de l'application de la formule de dotation de personnel est moindre que 1 FTE, les services d'un ou d'une bibliothécaire professionnel(le) peuvent être assurés selon les méthodes suivantes :

- Bibliothécaire contractuel(le) : Un arrangement contractuel écrit peut être négocié avec un ou une bibliothécaire contractuel(le) pour assurer la permanence de la direction et des services à un niveau approprié aux besoins de la clientèle, des programmes et services de l'établissement et aux buts et objectifs de la bibliothèque.

- Bibliothécaire itinérant(e) ou de secteur : Dans certaines régions, des établissements de soins de santé plus importants ou des bibliothèques académiques peuvent offrir les services d'un ou d'une bibliothécaire itinérant(e) ou de secteur. Le ou la bibliothécaire itinérant(e) ou de secteur effectue des visites selon un calendrier préétabli dans les établissements de soins de santé afin d'offrir des services de recherche et de récupération d'information et d'assurer que les ressources et les services offerts sur les lieux répondent effectivement aux besoins de la clientèle.

- Convention de coentreprise ou de services diversifiés : Les services d'un ou d'une bibliothécaire, de même que le mandat de fourniture de ressources d'information peuvent être partagés par les établissements sous réserve d'une convention de coentreprise ou de services diversifiés.

\section{Annexe 2 - Glossaire}

\section{Bibliothécaire itinérant(e) ou de secteur}

Un ou une bibliothécaire professionnel(le) qui offre des services selon un calendrier préétabli à plus d'une bibliothèque de petits établissements de santé. Cette personne peut faire appel aux collections et services de bibliothèques de sciences de la santé plus importantes pour assurer les services aux petites bibliothèques.

\section{Circulation}

Fonctions de bibliothèques qui consistent au prêt de documents pour utilisation à l'extérieur de la bibliothèque.

\section{Client ou clientèle}

Tout individu ou toute bibliothèque qui bénéficie de services de bibliothèque et d'information. Les clients peuvent être internes à l'établissement ou externes.

\section{Consortiums}

Un groupe de bibliothèques qui on convenu formellement d'un partage coopératif des ressources.

\section{Collection de base}

Une collection minimale de ressources d'information qui font autorité dans un domaine quelconque ou particulières à un type de bibliothèque.

\section{Sensibilisation aux mises à jour}

Services conçus en vue de maintenir à jour les clients sur les nouveaux développements et les nouvelles publications dans leur domaine particulier d'intérêt.

\section{Fourniture de documentation}

Fourniture de ressources de bibliothèque et d'information demandées par les clients. La fourniture de documents peut inclure la circulation, les services de photocopie et les prêts entre bibliothèques.

\section{Connaissance en information}

La connaissance en information est la capacité de reconnaître quand l'information est nécessaire, de la localiser, de l'évaluer et de l'utiliser efficacement.

\section{Prêt entre bibliothèques}

Le prêt entre bibliothèques est un mécanisme d'emprunt ou de prêt de documents originaux entre des bibliothèques qui travaillent en collaboration.

\section{Information basée sur les connaissances}

L'information basée sur les connaissances comprend les systèmes et les services destinés à aider les professionnels de la santé à acquérir les connaissances et les compétences nécessaires au maintien et à l'amélioration de leurs compétences, qui servent au processus décisionnel clinique, de gestion et d'affaires, qui appuient l'amélioration du rendement et des activités destinées à réduire le risque pour les patients et qui répondent aux besoins de recherche. 


\section{Bibliothèque}

Un éventail complet de services et de ressources conçus sur mesure pour répondre aux besoins d'information d'un groupe particulier d'utilisateurs, organisés de façon à en faciliter l'accès et qui sont sous la direction d'un ou d'une bibliothécaire professionnel(le).

\section{Évaluation des besoins}

Un processus systématique conçu dans le but de déterminer le besoin de services particuliers ou de types d'information destinés à un groupe d'utilisateurs. Le processus peut prendre la forme de sondages, de groupes de réflexion et d'entrevues.

\section{Bibliothécaire professionnel(le)}

Un ou une bibliothécaire professionnel(le) a obtenu une maîtrise d'un programme agréé par l'American Library Association (ALA) ou reconnu par l'ALA ou par un organisme national approprié.

\section{Annexe 3 - Lectures recommandées}

\section{Administration et organisation}

Eagleton KM. Quality assurance in Canadian hospital libraries - the challenge of the eighties. Health Libr Rev. 1988;5:145.

Forsman RB. Administration and management in health sciences libraries. Lanham, Md.: Medical Library Association and the Scarecrow Press; 2000.

Holst R, Phillips SA, Bensing KM, Medical Library Association. The Medical Library Association guide to managing health care libraries. New York: Neal-Schuman Publishers; 2000.

\section{Répercussions}

Burton JE. The impact of medical libraries and literature on patient care in New Zealand. Bull Med Libr Assoc. 1995 Oct;83(4):425-430.

Freeth DS, Weist A, Roberts CM. Provision of an electronic library at the clinical frontline: evaluation of impact on hospital medical staff. Hosp Med. 2001 Jan;62(1):43-45.

Haynes RB, McKibbon KA, Walker CJ, Ryan N, Fitzgerald D, Ramsden MF. Online access to MEDLINE in clinical settings. A study of use and usefulness. Ann Intern Med. 1990 Jan 1;112(1):7884.

Klein MS, Ross FV, Adams DL, Gilbert CM. Effect of online literature searching on length of stay and patient care costs. Acad Med. 1994 Jun;69(6):489-495.

Marshall JG. The impact of the hospital library on clinical decision making: the Rochester study. Bull Med Libr Assoc. 1992 Apr;80(2):169-178.

Moore ME, Garrison S, Hayes B, McLendon W. Reinventing a health sciences digital library - organizational impact. Med Ref Serv Q. 2003 Winter;22(4):75-82.

O'Connor P. Determining the impact of health library services on patient care: a review of the literature. Health Info Libr J. 2002 Mar;19(1):1-13.

Richwine MP, McGowan JJ. A rural virtual health sciences library project: research findings with implications for next generation library services. Bull Med Libr Assoc. 2001 Jan;89(1):37-44.
Sherwill-Navarro PJ, Wallace AL. Research on the value of medical library services: does it make an impact in the health care literature? J Med Libr Assoc. 2004 Jan;92(1):34-45.

Weightman AL, Williamson J, Library \& Knowledge Development Network (LKDN) Quality and Statistics Group. The value and impact of information provided through library services for patient care: a systematic review. Health Info Libr J. 2005 Mar;22(1):4 25.

Weightman A,L., Williamson J. The value and impact of information provided through library services for patient care: a systematic review. Health Information and Libraries Journal. 2005;22(1):4-25.

\section{Sécurité des patients}

Institute of Medicine. Committee on Quality of Health Care in America. Crossing the quality chasm : a new health system for the 21st century. Washington, D.C.: National Academy Press; 2001.

Ludwig L, Starr S. Library as place: results of a delphi study. J Med Libr Assoc. 2005 Jul;93(3):315-326.

Williams L, Zipperer L. Improving access to information: librarians and nurses team up for patient safety. Nurs Econ. $2003 \mathrm{Jul}-$ Aug;21(4):199-201.

Zipperer L. Clinicians, librarians and patient safety: opportunities for partnership. Qual Saf Health Care. 2004 Jun;13(3):218-222.

Zipperer L, Sykes J. The role of librarians in patient safety: gaps and strengths in the current culture. J Med Libr Assoc. 2004 Oct;92(4):498-500.

\section{Dotation de personnel}

Competency Profile. Information Resources Management specialists in Archives, Libraries and Records Management: A comprehensive Cross-Sectoral competency Analysis. 1999 April 1999.

Crosby O. Librarians: Information experts in the information age. OOQ. 2001(Winter 2000-01):2.

Medical Library Association. Medical Librarianship: A Career Beyond the Cutting Edge.

Stanley E. Competencies for hospital librarians. Natl Netw. 2000 Apr;24(4):12-3, 20.

\section{Normes}

Australia Library and Information Association. Guidelines for Australian Health Libraries. 2000; Accessible à http://www.alia.org. au/policies/health.libraries.html. Accessed January 13, 2006.

Flower MA. Toward hospital library standards in Canada. Bull Med Libr Assoc. 1978 Jul;66(3):296-301.

Fowler C, Trinder V. Accreditation of Library and Information Services in the Health Sector. A Checklist to Support Assessment. Health Libraries and Information Confederation (HeLICon) Working Group. 2002; Accessible à http://www.nelh.nhs.uk/librarian/ Accreditation_Checklist_2nd_Edition_2002.pdf. Accessed January 13, 2006.

Hassig RA, Balogh L, Bandy M, Doyle JD, Gluck JC, Lindner KL, et al. Standards for hospital libraries 2002 with 2004 revisions. J Med Libr Assoc. 2005 Apr;93(2):282-283.

Lucey N. Standards for Irish Healthcare Library and Information Services. 2nd ed. Dublin, Ireland: Library Association of Ireland; 2005. Accessible à http://hrb.tinderbox.ie/archives/Standards_ cover.pdf. 